receiving tumour necrosis factor inhibitors (TNFi) while $40 \%$ were on corticosteroids (mean daily dose: $4.7 \mathrm{mg}$ ). Despite these therapies, $43 \%$ of patients had active disease (DAS28-ERS>3.2); 34\% moderate (MDA, DAS28-ESR=3.2-5.1) and $9 \%$ high (HDA, DAS28-ESR $>5.1$ ) disease activity. During the 1 year observation period, among the group of patients with MDA who were only on csDMARDs, $15 \%$ started a bDMARD while among those on bDMARDs, $11 \%$ switched to another bDMARD. The respective rates of starting a bDMARD (in those on csDMARDs) or switching to another bDMARD (in those on bDMARDs), were much higher for patients in the HDA group ( $41 \%$ and $32 \%$ respectively, $p<0.001$ for both groups). At the end of the 1 st year, the proportion of patients on TNFi and corticosteroids was $57 \%$ and $32 \%$, respectively. Overall, despite a decrease in the DAS28-ESR score (from $3.2 \pm 1.2$ to $2.9 \pm 1.3, p<0.001), 37 \%$ of patients had still active disease $(6 \%$ improvement after 1 year; MDA: 30\%, HDA: $7 \%$ ).

Conclusions: In a large, real life, RA cohort with almost half of patients on bDMARD-based therapies, more than one third of patients had still active disease at the end of the 1st year of follow-up. These findings could be explained in part by the low rate of bDMARD initiation or switching in this cohort or they could indicate the limitations of current therapeutic approaches in RA patients with longstanding disease in Greece.

Acknowledgements: Supported by grants from the Greek Rheumatology Society and Professional Association of Rheumatologists.

Disclosure of Interest: None declared

DOI: 10.1136/annrheumdis-2018-eular.6944

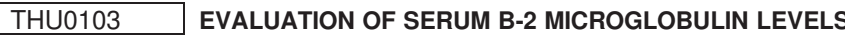 AND ITS RELATIONSHIP WITH DISEASE ACTIVITY IN RHEUMATOID ARTHRITIS}

A.B. Kelesoglu Dincer, M. Torgutalp, E.G. Aydemir Gülöksüz, M.E. yayla, E. Uslu Yurteri, I.E. Okatan, S. Sezer, G. Kinikli, T.M. Turgay, A. Ateș. Rheumatology, Ankara University Faculty of Medicine Rheumatology Department, Ankara, Turkey

Background: $\beta-2$ microglobulin ( $\beta 2 M G$ ) is produced and secreted from $T$ and $B$ lymphocytes. In autoimmune diseases, due to lymphocyte activation and proliferation, serum $\beta 2 M G$ level is expected to be higher than normal. In a few studies, increased serum and synovial fluid $\beta 2 M G$ levels in rheumatoid arthritis (RA) were reported.

Objectives: The aim of this study was to evaluate the concentration of $\beta 2 \mathrm{MG}$ in peripheral blood in RA patients and to show its relationship with disease activity. Methods: In this study, 121 RA patients and 50 sex and age matched healthy controls were enrolled. Patients who had other inflammatory diseases or any kind of malignancy were excluded.

Results: The mean age of RA patients (97 female and 24 male) were 55 years ( \pm 10.9 years) and mean disease duration was 9 years (min: 6 months, max: 42 years). The demographic data of patients and healthy controls are shown in table 1. There was a statistically significant difference between haemoglobin, erythrocyte sedimentation rate and C-reactive protein levels between two groups $(p<0.001$, $p<0.001, p=0.001$ respectively). Serum $\beta 2 M G$ levels were higher in RA group than healthy controls. $(p<0.001)$ When RA patients were grouped according to disease activity as remission to low disease activity(DAS28-ESR<3.2) $(n=65)$ and moderate to severe disease activity(DAS28-ESR $\geq 3.2)(n=56)$, the serum $\beta 2 M G$ levels were higher in DAS28-ESR $\geq 3.2$ group than in DAS28-ESR $<3.2$ group $(p=0.003)$. The difference between serum $\beta 2 M G$ levels were statistically significant in low and high disease activity groups when grouped both according to DAS-ESR and DAS-CRP $(\mathrm{p}=0.003$ and $\mathrm{p}=0.006$, respectively). The seropositivity between two groups were similiar $(p=0.385$ ), although serum ESR, CRP levels and the rate of morning stiffness were significantly higher in high disease activity group. The results were shown in table 2. According to Spearmann correlation test, the serum $32 \mathrm{MG}$ level and DAS-ESR and DAS-CRP were positively correlated which were both statistically significant. $(r=0.378, p<0.001$ and $r=0.324, p<0.001$ respectively).

Abstract THU0103 - Table 1. Baseline characteristics of RA patients and healthy controls

\begin{tabular}{lccc}
\hline Characteristics & $\mathrm{RA}(\mathrm{n}=121)$ & $\begin{array}{c}\text { Control } \\
(\mathrm{n}=50)\end{array}$ & p value \\
\hline $\begin{array}{l}\text { Female Sex, } \\
(\%)\end{array}$ & $97(80.2)$ & $35(70.0)$ & 0.150 \\
Age, years & $55.0 \pm 10.9$ & $52.6 \pm 10.6$ & 0.183 \\
WBC & 7736 & $7170 \pm 1759$ & 0.139 \\
& \pm 3177 & & \\
Hb & $12.6 \pm 1.5$ & $14.1 \pm 1.7$ & $<0.001$ \\
Thrombocyte & 290.4 & $279.7 \pm 65.2$ & 0.441 \\
& \pm 88.9 & & \\
ESR & $24.3 \pm 17.0$ & $12.2 \pm 8.2$ & $\mathbf{0 . 0 0 1}$ \\
CRP (IQR) & $2.5(4.7)$ & $6.1(10.7)$ & $\mathbf{0 . 0 0 1}$ \\
$\beta 2-$ microglobulin & $2.93 \pm 1.20$ & $2.21 \pm 0.54$ & $<\mathbf{0 . 0 0 1}$ \\
DAS28-ESR & $3.30 \pm 1.16$ & & \\
DAS28-CRP & $3.60 \pm 1.39$ & & \\
\hline
\end{tabular}

Abstract THU0103 - Table 2. Baseline characteristics of RA patients

\begin{tabular}{lccc}
\hline & $\begin{array}{c}\text { DAS28-ESR } \leq 3.2 \\
(\mathrm{n}=65)\end{array}$ & $\begin{array}{c}\text { DAS28-ESR>3.2 } \\
(\mathrm{n}=56)\end{array}$ & p value \\
\hline Female Sex, $\mathrm{n}(\%)$ & $48(73.8)$ & $29(87.5)$ & 0.060 \\
Age, years & $53.4 \pm 11.3$ & $56.9 \pm 10.1$ & 0.079 \\
ESR & $17.8 \pm 12.3$ & $31.9 \pm 18.6$ & $<0.001$ \\
CRP (IQR) & $4.0(7.9)$ & $10.2(12.6)$ & $<0.001$ \\
B2-microglobulin & $2.62 \pm 0.87$ & $3.29 \pm 1.42$ & $\mathbf{0 . 0 0 3}$ \\
DAS28-ESR & $2.42 \pm 0.59$ & $4.31 \pm 0.78$ & $<0.001$ \\
DAS28-CRP & $2.67 \pm 0.61$ & $4.67 \pm 2.26$ & $<0.001$ \\
Morning stiffness, $\mathrm{n}(\%)$ & $13(20)$ & $24(42.9)$ & $\mathbf{0 . 0 0 7}$ \\
\hline
\end{tabular}

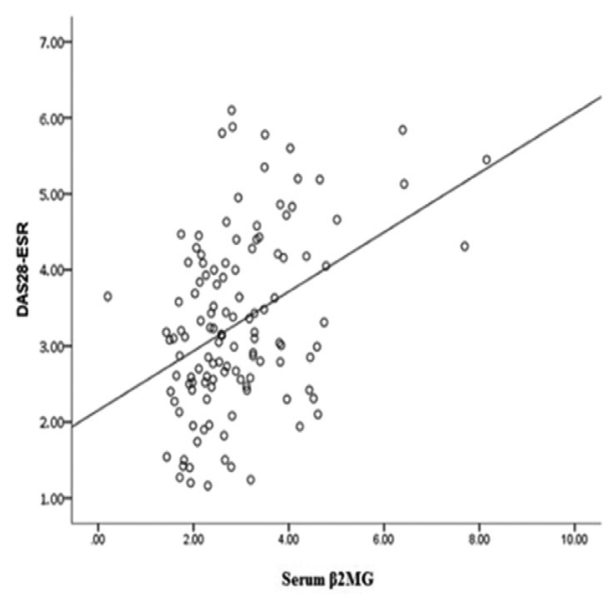

Abstract THU0103 - Figure 1. The figüre that shows the relationship of serum $\beta 2 M G$ with DAS28-ESR

Conclusions: Our results show that serum $\beta 2 M G$ concentration increase in RA and is higher in active patients. It can be concluded that serum $\beta 2 M G$ may be an appropriate parametre to monitor disease activity in rheumatoid arthritis.

Disclosure of Interest: None declared

DOI: 10.1136/annrheumdis-2018-eular.4258

\section{THU0104 THE TEMPORAL PROFILE OF ANTIBODIES DIRECTED AGAINST POST-TRANSLATIONAL MODIFICATIONS VARIES ACCORDING TO ISOTYPE AND TARGET IN PATIENTS WITH NEW-ONSET RHEUMATOID ARTHRITIS}

B. Dyke $^{1}$, M. Juarez ${ }^{2}$, H. Bang ${ }^{3}$, C.D. Buckley ${ }^{1}$, A. Filer ${ }^{1}$, K. Raza ${ }^{1}{ }^{1}$ University of Birmingham, Birmingham; ${ }^{2}$ UCB Pharma, Slough, UK; ${ }^{3}$ Orgentec Diagnostika GmBH, Mainz, Germany

Background: Autoantibodies directed against antigens with post-translational modifications (PTMs), such as citrullination (ACPA), are a hallmark of rheumatoid arthritis (RA) ${ }^{1}$. ACPA titres increase prior to disease onset, but are thought to be relatively stable after symptomatic inflammation is established ${ }^{2}$. The temporal profile of antibodies against acetylated (AAPA) and carbamylated (ACarPA) peptides has not been so comprehensively characterised following the onset of joint swelling ${ }^{3}$

Objectives: We aimed to track serum levels of anti-PTM antibodies over 18 months in patients with newly-presenting RA in our prospective observationa cohort.

Methods: Patients with treatment-naïve inflammatory arthritis donated serum at baseline, 6 and 18 months. 103 patients satisfying ACR/ ${ }^{\text {EULAR } 2010}$ criteria for RA underwent testing for $\lg G$ and $\lg A$ antibodies against peptides with citrulline (ACPA), carbamylated lysine (ACarPA), and acetylated lysine (AAPA) PTMs using ELISA as previously described ${ }^{4}$.

Results: $57 \%$ of participants were female, and $48 \%$ and $50 \%$ patients were antiCCP2 or rheumatoid factor positive respectively. Mean age was 56 years (s.d. 15.2), symptom duration 55 days (s.d 22.4), and DAS28CRP 4.4 (s.d. 1.3) at enrolment.

Comparing baseline and 18 month median antibody levels measured by optical density, a decrease was observed over time for $\lg \mathrm{G}(0.26$ vs $0.17, \mathrm{p}<0.0001)$ and $\lg$ A APA (0.23 vs $0.09, p<0.0001)$, as well as both $\lg G$ and $\lg A$ antibodies against citrullinated peptides ( 0.47 vs $0.40, p<0.0001$ and 0.16 vs $0.12, p<0.0001$ respectively). Significant reductions occurred between baseline and 6 months for both $\lg G(p<0.0001)$ and $\lg A(p<0.0001)$ AAPA antibodies, but not between 6 and 
18 month timepoints after correction for multiple testing. A similar pattern was observed for IgA ACPA antibody levels, although IgG ACPA levels did not vary significantly comparing baseline with 6 , and 6 with 18 month timepoints. Antibody levels were not influenced by exposure to therapy. Patients seropositive for antiCCP2 at baseline demonstrated less labile antibody levels, with the exception of sustained increases in IgG ACarPA reactivity compared with seronegative individuals. Abstract THU0104 - figure 1. demonstrates bidirectional changes in antiPTM antibody reactivity at the patient level.
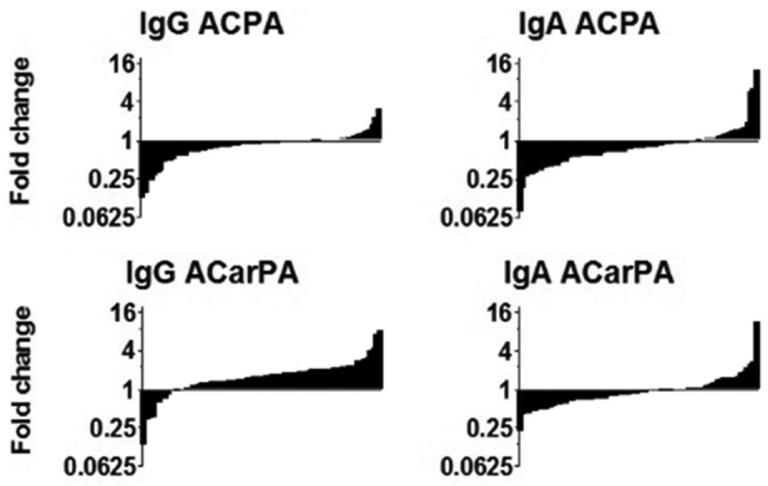

IgG AAPA

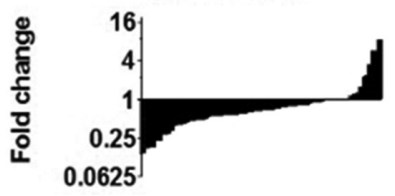

IgA AAPA

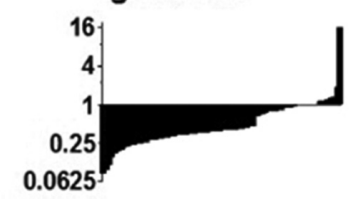

Abstract THU0104 - Figure 1. Fold change in anti-PTM autoantibody levels between baseline and 18 months in patients with newly-presenting rheumatoid arthritis $(n=130)$

Conclusions: Median AAPA and, to a lesser extent, ACPA levels fell over time, regardless of therapy. This was most marked for the $\lg A$ isotype. Differential isotype effects may represent maturation of the autoantibody repertoire from mucosal IgA antibodies involved in the breach of tolerance. Lability of AAPA levels may reflect the relative reversibility of acetylation of a lysine amino acid residue by comparison with citrullination and carbamylation modifications. By contrast, and as observed previously ${ }^{3}$, IgG ACarPA levels increased over time, perhaps reflecting the tendency of the human proteome to accrue carbamylation modifications due to ageing, and metabolic or inflammatory stress.

\section{REFERENCES:}

[1] Trouw LA, et al. Nat Rev Rheumatol 2017;13: 331-9.

[2] Modi S, et al. Clin Exp Immunol 2013;173(1):8-17.

[3] Spinelli FR, et al. J Rheum Dis Treat 2015;1:13.

[4] Juarez M, et al. Ann Rheum Dis 2016;75:1099-1107.

Acknowledgements: This work was funded via an EU FP7 grant (EuroTeam). $\mathrm{KR}$ is funded by the Birmingham NIHR Biomedical Research Centre.

Disclosure of Interest: None declared

DOI: 10.1136/annrheumdis-2018-eular.1561

\section{THU0105 RELATIONSHIP BETWEEN DAS28 CATEGORIES AND RAID PATIENT REPORTED OUTCOME IN RHEUMATOID ARTHRITIS; SIGNIFICANT ADVANTAGE OF TARGETING DAS REMISSION}

C. Smith ${ }^{1}$, M. Sharif ${ }^{1}$, A.A. Prideaux ${ }^{2}$, P.D. Kiely ${ }^{1} .{ }^{1}$ Rheumatology, ST Georges University Hospitals NHS Foundation Trust, London; ${ }^{2}$ School of Clinical Medicine, University of Cardiff, Cardiff, UK

Background: EULAR/ACR guidelines recommend remission or low DAS28 as the treat to target goal for patients with rheumatoid arthritis (RA). The DAS28 is a composite score derived from objective (swollen joint count and ESR/CRP) and subjective (tender joint count and patient global) measures of disease activity, restricted to 28 joints. It has been criticised as not being representative of the whole patient or completely allied to patient experience. Alternative patient reported outcomes (PROM) have been developed, including the rheumatoid arthritis impact of disease (RAID) which is a self-reported index which assesses seven domains by visual analogue scale: pain, disability, fatigue, sleep, coping, physical and emotional well-being. Responses are weighted differently producing a final score from $0-10$ and a score $<2$ is considered a patient-acceptable status. Objectives: Given uncertainty over the necessity to aim for DAS remission (RDAS) as opposed to low DAS (LDAS) as a treatment target, we sought to explore the relation between DAS outcomes and RAID scores in routine clinical practice.

Methods: RA patients attending for routine review in the outpatient clinic at St George's Hospital were assessed by a physician associate between June 2016 and September 2017. DAS28 CRP and ESR scores were recorded and RAID questionnaires completed by patients and calculated using the on-line EULAR tool. Data were analysed on Excel for summary statistics and Spearman correlation coefficient and socscistatistics.com for Mann-Whitney $U$ tests.

Results: 117 RA patients were assessed, 84\% female, mean age 59.6 years, $77 \%$ RF positive and $85 \%$ ACPA positive. The prevalence of DAS28 ESR categories was RDAS $(\leq 2.6) n=57(49 \%)$, LDAS $(2.6-3.2) n=17(14.5 \%)$, moderate (MDAS 3.21-5.1) $n=35$ (30\%), high (HDAS $\geq 5.1) n=8(6.5 \%)$. RAID scores correlated strongly with patient global $(r=0.62)$, DAS28 CRP $(r=0.58)$ and DAS28 ESR $(r=0.54)$ but poorly with tender joint count $(r=0.32)$, swollen joint count $(r=0.10)$, ESR $(r=0.13)$ and CRP $(r=0.09)$. The mean RAID score in DAS28 ESR categories was RDAS 2.49, LDAS 3.77, M+HDAS 5.92, see figure 1, box and whisker plots. RAID scores were significantly different (Mann-Witney $U$ ) between M+HDAS verrsus RDAS $(p<0.0001), M+H D A S$ versus LDAS $(p=0.0048)$ and also between RDAS versus LDAS ( $p$ 0.013). Similar significant differences in RAID scores were found with DAS28 CRP categories. There were no significant differences in RAID scores between RF positive versus negative or ACPA positive versus negative patients.

Of 30 patients with RAID <2, DAS28 ESR was <2.6 in $27(90 \%)$ and $<3.2$ in 29 $(97 \%)$. Of 74 patients with DAS28 ESR $<3.2$, RAID was $>2$ in $45(61 \%)$ with fatigue followed by sleep being the worst scoring domains.

\section{RAID SCORE}

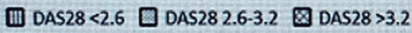

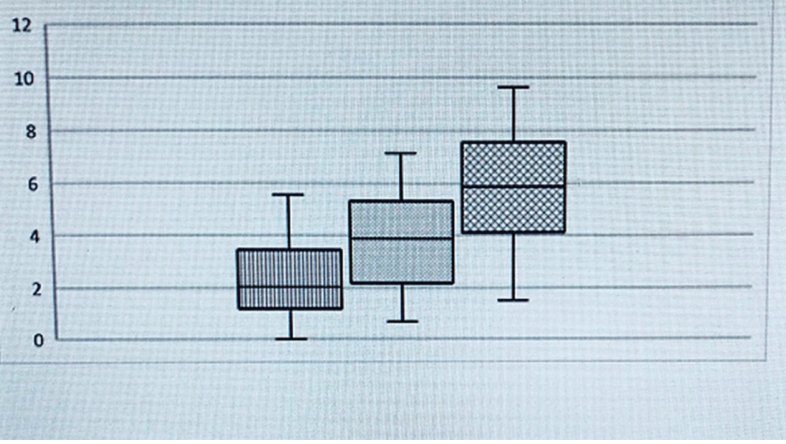

Conclusions: RAID scores strongly correlate with patient global and total DAS28 (ESR or CRP) scores, and are significantly different between all DAS categories, including RDAS versus LDAS. Patients with RAID $<2$ are almost all at LDAS target, but high numbers at LDAS have unacceptable RAID, largely driven by high fatigue and sleep scores. This suggests that achieving RDAS has significant benefit over LDAS from a PROM perspective, and attention should be paid to fatigue and sleep if RAID is unacceptable in LDAS patients.

Disclosure of Interest: None declared

DOI: 10.1136/annrheumdis-2018-eular.4313

\section{THU0106 CONVERTING PATIENT-REPORTED OUTCOME MEASURES OF FATIGUE AND PAIN TO PROMIS SCORES: DATA FROM PHASE 3 BARICITINIB RHEUMATOID ARTHRITIS TRIALS}

C.O. Bingham III ${ }^{1}$, C. Gaich ${ }^{2}$, A.M. DeLozier ${ }^{2}$, A. Quebe $^{2}$, L. Sun ${ }^{2}$, S. Otawa ${ }^{2}$, J. Pope ${ }^{3}$. 1 Johns Hopkins Univ, Baltimore; ${ }^{2}$ Eli Lilly and Company, Indianapolis, USA; ${ }^{3}$ Joseph's Health Care, London, Alberta, Canada

Background: Fatigue and pain in patients (pts) with RA are often measured with the Functional Assessment of Chronic Illness Therapy-Fatigue (FACIT-F) and the Medical Outcomes Study Short-Form-36 (SF-36). Patient-Reported Outcomes Measurement Information System (PROMIS) was developed using a populationcalibrated T-score metric (Mean 50, SD 10). Crosswalk tables were developed linking legacy instruments to PROMIS instruments, including Fatigue and Pain 